\title{
INTERNATIONAL JOURNAL OF \\ Clinical features and prognostic factors of cervical villoglandular adenocarcinoma
}

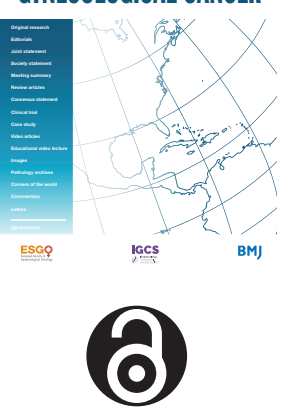

OPEN ACCESS

- Additional material is published online only. To view please visit the journal online (http://dx.doi.org/10.1136/ijgc2020-002044).

For numbered affiliations see end of article.

\section{Correspondence to}

Xiaojian Yan, Department of Gynecology, The First Affiliated Hospital of Wenzhou Medical University, Zhejiang 325000, China; yxjbetter2016@hotmail. com and Dr Chunlin Chen, Department of Obstetrics and Gynecology, Nanfang Hospital, Southern Medical University, Guangzhou 510515, China; ccl1@smu.edu.cn

J-HC, HD, X-BY and H-WZ contributed equally.

Received 14 September 2020 Revised 23 December 2020 Accepted 29 December 2020 Published Online First 19 February 2021

\section{Check for updates}

(C) IGCS and ESG0 2021. Re-use permitted under CC BY-NC. No commercial re-use. Published by BMJ.

To cite: Chen J-H, Duan H Yu X-B, et al. Int J Gynecol

Cancer 2021;31:512-517.

Jia-Hua Chen, ${ }^{1}$ Hui Duan, ${ }^{2}$ Xiao-Bo Yu, ${ }^{1}$ Hong-Wei Zhao, ${ }^{3}$ Xu Chen, ${ }^{4}$ Pengfei Li, ${ }^{2}$ Zhi-Qiang Li, ${ }^{2}$ Bing-Xin Li, ${ }^{1}$ Lu-Yao Pan, ${ }^{1}$ Xiaojian Yan (D),${ }^{1,5}$ Chunlin Chen ${ }^{2}$

\section{HIGHLIGHTS}

- Villoglandular adenocarcinomas rarely have lymph node involvement (5\%) or lymphovascular invasion (3\%).

- No death or recurrence occurred in patients with villoglandular adenocarcinoma during the follow-up period of 50.2 months.

- The 5-year disease-free survival of patients with villoglandular adenocarcinoma was significantly better than of patients with other types of cervical adenocarcinoma

\section{ABSTRACT}

Background Villoglandular adenocarcinoma is a rare sub-type of cervical adenocarcinoma.

Objective To analyze the clinicopathological features and evaluate the prognosis of patients with villoglandular adenocarcinoma of the cervix.

Methods Patient characteristics, procedure, pathology, and surgical outcomes were retrospectively reviewed in patients with villoglandular adenocarcinoma between November 2006 and June 2019 from multiple centers in China. In order to explore the difference between villoglandular adenocarcinoma and routine adenocarcinoma, patients (FIG0 2009 stage IA1-IB2) who had complete data during the same time period were included.

Results A total of 60 patients with villoglandular adenocarcinoma and 104 with standard adenocarcinoma were included. The median age of the patients with villoglandular adenocarcinoma was 42 years (range 27-68). The most common 2009 FIG0 stage was IB1 in $39(65 \%)$ patients with villoglandular adenocarcinoma. A total of 23 patients underwent laparoscopic surgery (two total hysterectomies, 21 radical hysterectomies) and the other 37 patients underwent laparotomy (three total hysterectomies, 34 radical hysterectomies). A total of 56 patients underwent lymphadenectomy and three (5.4\%) had positive lymph nodes. Fifteen (25\%) patients had one or both ovaries preserved. Seven patients were lost to follow-up. The median follow-up time for the entire group was 50.2 months (range 5.1-154.6). No deaths or recurrences occurred. Excluding six patients with FIG0 2009 stage II, the 5-year disease-free survival of the 47 patients with villoglandular adenocarcinoma with FIGO 2009 stage I for whom there was follow-up, was significantly higher than that of the 104 patients with standard cervical adenocarcinoma ( $100 \%$ vs $92.2 \%$, logrank $p=0.039)$. However, the 5 -year overall survival of the two groups did not differ ( $100 \%$ vs $95.7 \%$, log-rank $\mathrm{p}=0.11$ ).

Conclusion Villoglandular adenocarcinoma has a favorable prognosis. Further studies are needed to provide more details of treatment strategies and prognosis.

\section{INTRODUCTION}

Cervical cancer is one of the most common malignant tumors in the female reproductive system in developing countries. A total of 13240 new cases and 4170 deaths were estimated in the USA in 2019. ${ }^{1}$ In 2018, there were 570000 new cases of cervical cancer, which represented $6.6 \%$ of all female carcinomas in the world. ${ }^{2}$ The incidence of squamous cell carcinoma has declined significantly and the incidence of cervical adenocarcinoma continuously increased from $5 \%$ to $25 \%$ in the past decades. ${ }^{3-5}$ Cervical adenocarcinoma is generally associated with lymph node metastasis and distant metastasis, which may lead to poor prognosis. ${ }^{6-9}$

Villoglandular adenocarcinoma is a rare sub-type of cervical adenocarcinoma and it was first described in 1989 by Young and Scully. ${ }^{10}$ The International Endocervical Adenocarcinoma Criteria and Classification (IECC) declared that villoglandular adenocarcinoma is an human papillomavirus (HPV)-associated adenocarcinoma. ${ }^{11}$ The incidence of villoglandular adenocarcinoma is between $4 \%$ and $8.8 \%,{ }^{12}{ }^{13}$ which is lower than for cervical adenocarcinoma. ${ }^{14-16}$ Villoglandular adenocarcinoma tumors have a lower risk of deep infiltration, lymphovascular invasion, and lymph node metastasis. ${ }^{15} 17$ The majority of studies suggest that the long-term prognosis of villoglandular adenocarcinoma is considered favorable. ${ }^{16-18}$

The standard surgery for patients with International Federation of Gynecology and Obstetrics (FIG0 2009) stage IA1 combined with lymphovascular invasion and stage IA2-IIA1 cervical adenocarcinoma is radical hysterectomy and pelvic lymph node assessment. ${ }^{719}$ In this study, the clinical and pathological characteristics of patients with villoglandular adenocarcinoma in multiple centers in China were reviewed and the risk factors were evaluated. We also compared the outcomes of patients with villoglandular carcinoma with those of patients with standard adenocarcinoma in the same period. 


\section{METHODS}

Patients with adenocarcinoma between November 2006 and June 2019 were retrospectively reviewed. Clinical characteristics, HPV testing, pathological features, treatment, and prognosis were analyzed. Inclusion criteria of patients with villoglandular adenocarcinoma were as follows: villoglandular adenocarcinoma confirmed by pre-operative biopsies and/or post-operative pathological examination. Pathological diagnostic criteria for villoglandular adenocarcinoma were as follows: exophytic pattern of growth with long and slender papillary structures, mild to moderate cellular atypia, and scattered mitotic figures. All specimens were reviewed by at least two pathologists and audited by a senior pathologist. Exclusion criteria were the presence of other malignancy from either villoglandular carcinoma or standard adenocarcinoma and incomplete medical records.

In order to explore the difference between villoglandular adenocarcinoma and standard adenocarcinoma with FIGO 2009 stage I, a total of 104 patients with standard cervical adenocarcinoma (FIGO 2009 stage I) were screened in the same period. The establishment of the cervical cancer database was reviewed by the ethics committee of Nanfang Hospital, Southern Medical University (ethics number NFEC-2017-135).

Statistical Product and Service Solutions (SPSS) 25.0 statistical software was used to analyze the data. A t-test was adopted for measurement of data. Enumeration data were evaluated using the chi-square test and Fisher exact test. The survival patterns of patients with villoglandular adenocarcinoma and patients with standard adenocarcinoma were shown graphically using KaplanMaier survival curves. Groups were compared by the log-rank test.

\section{RESULTS}

A total of 1515 patients with adenocarcinoma were screened and 60 patients with villoglandular adenocarcinoma (4\%) were included in our analysis (Online supplemental table 1). The median age was 42 years (range 27-68). The most common symptoms were irregular vaginal bleeding or post-coital bleeding $(41.9 \%)$. The most common tumor growth pattern was exophytic type (67.9\%). The 2009 FIGO stage distribution was 4 patients stage IA2, 39 patients stage IB1, 11 patients stage IB2, 5 patients stage IIA1, and 1 patient stage IIA2. A total of seven patients were lost follow-up (Figure 1). The mean maximum diameter of the tumor was $2.12 \pm 1.78 \mathrm{~cm}$ and tumors $\geq 2 \mathrm{~cm}$ accounted for $71.7 \%$ (Table 1 ).

In the laparoscopic surgery group $(n=23)$, two patients underwent laparoscopic total hysterectomy without pelvic lymphadenectomy and 21 patients underwent laparoscopic radical hysterectomy with pelvic lymphadenectomy. In the laparotomy surgery group $(n=37)$, three patients underwent total hysterectomy and only one underwent pelvic lymphadenectomy. The other 34 patients underwent laparotomy radical hysterectomy group with pelvic lymphadenectomy. The median follow-up was 38.8 months (range 13-85.2) for patients who had laparoscopic surgeries and 56.5 months (range 5.1-154.6) for those who underwent open surgeries (Table 1). A total of 56 patients underwent lymphadenectomy and three (5.4\%) had positive lymph nodes. A total of 27 (45\%) patients received adjuvant therapy (Online supplemental table 1).

Eighteen of 20 patients had positive-HPV results and nine of 13 patients had abnormal cervical cytology screening (Table 1). In a total of $38.3 \%$ of patients the preoperative diagnosis of villoglandular adenocarcinoma was consistent with the post-operative result. Villoglandular adenocarcinomas were found to be primarily well differentiated (grade l/II , 97.1\%), rarely to have lymphovascular space invasion (3.3\%), and mainly having superficial cervical interstitial infiltration $(\leq 1 / 2,71.7 \%)$. A total of $6.7 \%$ of patients had uterine infiltration and $5.4 \%$ of patients had lymph node metastasis (Table 1). In this study, $75 \%$ patients were found to be p16 positive and the Ki67 index was increased in $75 \%$ of patients (Online supplemental table 2).

A total of 15 patients preserved unilateral/bilateral ovaries. These patients were younger (39.6 vs 45.2 years, $p=0.024$ ) and more underwent simple hysterectomy ( $26.7 \%$ vs $2.2 \%, p=0.015$ ) in comparison with those who had oophorectomy. No tumor recurrence or metastasis occurred in these patients. Moreover, there was no statistical difference in pathological factors such as tumor differentiation, lymphatic vessel infiltration, interstitial infiltration depth, uterine infiltration, lymph node metastasis, parametrium, and vaginal resection margin infiltration $(p>0.05)$.

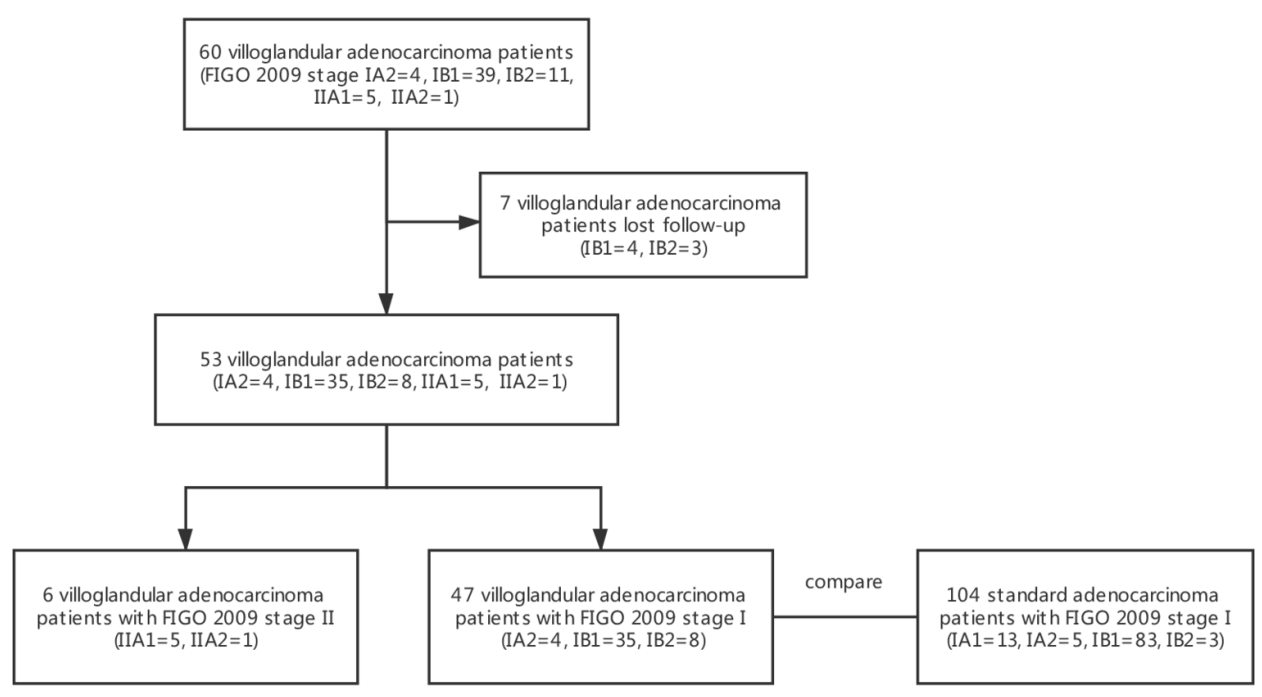

Figure 1 Flow diagram of patient selection. 


\section{Original research}

The clinicopathological features of 47 patients with villoglandular adenocarcinoma were compared with those of 104 patients with common adenocarcinoma (FIGO 2009 stage I). No significant difference was found in the rate of HPV infection between the two groups $(p=0.99)$. However, patients with villoglandular adenocarcinoma were more likely to have HPV16 sub-types $(70.6 \%$ vs $33.3 \%$, $\mathrm{p}=0.004$ ), whereas patients with cervical adenocarcinoma were more likely to have HPV18 sub-types $(17.6 \%$ vs $47.6 \%, p=0.023)$. In addition, patients with villoglandular adenocarcinoma were younger ( 43.8 vs 47.1 years, $p=0.029$ ) than patients with standard adenocarcinoma. Comparison of the villoglandular adenocarcinoma group with the group with standard adenocarcinoma showed that the proportion of pre-menopausal women was higher in the former $(81.8 \%$ vs $66.4 \%, p<0.001)$, rates of grade I were higher $(46.4 \%$ vs $17.6 \%, p=0.003$ ), and there was more superficial cervical infiltration $(70.0 \%$ vs $66.7 \%, p=0.004)$. Although more $\mathrm{FIGO}$ stage IB2 patients were included in the villoglandular adenocarcinoma group $(17.0 \%$ vs $2.9 \%, p=0.001)$, no recurrence or death occurred in the villoglandular adenocarcinoma group. In the standard adenocarcinoma group, six patients relapsed and three patients died of disease (Table 2). The 5-year disease-free survival of patients with villoglandular adenocarcinoma was significantly better $(100 \%$ vs $92.2 \%$, log-rank $p=0.039$ ) than that of patients with standard adenocarcinoma, but no significant differences in 5-year overall survival was noted between the two groups $(100 \%$ vs $95.7 \%$, log-rank $p=0.11$ ) (Figure 2). Analysis of disease-free survival and overall survival between the two groups with or without ovarian preservation showed no difference $(p>0.05)$. None of the patients who underwent simple hysterectomy relapsed (Figure 3).

\section{DISCUSSION}

\section{Summary of Main Results}

Our results demonstrate that patients with villoglandular adenocarcinoma have more favorable clinicopathologic features and prognosis than patients with standard cervical adenocarcinoma. No deaths or recurrence occurred even in patients who underwent laparoscopic surgery or more conservative surgery, such as simple hysterectomy or ovarian preservation, during the follow-up period of 50.2 months.

\section{Results in the Context of Published Literature}

The literature has reported that the age range of patients with villoglandular adenocarcinoma is between 33 and 52 years. ${ }^{5710121320-23}$ Our study reported that patients with villoglandular adenocarcinoma were younger than those with standard cervical adenocarcinoma and $81 \%$ were pre-menopausal women.

Villoglandular adenocarcinoma is associated with superficial invasion, well-differentiated tumor, uncommon lymphovascular invasion, and rare lymph node metastasis. Prior work reported the depth of stromal invasion of $64.3 \%$ patients to be less than $1 / 2{ }^{12}$ The lymph node metastasis rate of villoglandular adenocarcinoma is reported as between $2.7 \%$ and $11.7 \% .^{1324}$ Lower lymph node metastasis $(2.6 \%$ vs $14.2 \%, p=0.001)$ in villoglandular adenocarcinoma was reported than in standard adenocarcinoma. ${ }^{13}$

So far, at least 203 cases of villoglandular adenocarcinoma have been reported, with 12 recurrences and eight deaths. ${ }^{12}{ }^{13}$ Most had more than one risk factor, such as lymph
Table 1 Clinicopathological features and prognosis of 60 patients with villoglandular adenocarcinoma*

\begin{tabular}{|c|c|}
\hline Clinical features & Value \\
\hline Median (IQR) age & $42(27-68)$ \\
\hline Tumor size $\left(\right.$ mean $\left._{ \pm} \mathrm{SD}\right)$ & $2.12 \pm 1.78$ \\
\hline$<2 \mathrm{~cm}$ & $17(28.3)$ \\
\hline $2-3.9 \mathrm{~cm}$ & $25(41.7)$ \\
\hline$\geq 4 \mathrm{~cm}$ & $18(30.0)$ \\
\hline \multicolumn{2}{|l|}{ FIGO stage (2009) } \\
\hline IA2 & $4(6.7)$ \\
\hline IB1 & $39(65.0)$ \\
\hline IB2 & $11(18.3)$ \\
\hline$\| \mathrm{A} 1$ & $5(8.3)$ \\
\hline IIA2 & $1(1.7)$ \\
\hline \multicolumn{2}{|l|}{ Primary therapy } \\
\hline Surgery & $50(83.3)$ \\
\hline NACT & $7(11.7)$ \\
\hline NAIC & $3(5.0)$ \\
\hline \multicolumn{2}{|l|}{ Surgery } \\
\hline Radical hysterectomy & $55(91.7)$ \\
\hline Simple hysterectomy & $5(8.3)$ \\
\hline \multicolumn{2}{|l|}{ Surgical approach } \\
\hline Laparoscopy & $23(38.3)$ \\
\hline Laparotomy & $37(61.7)$ \\
\hline \multicolumn{2}{|l|}{ Adjuvant treatment } \\
\hline Radiotherapy & $2(3.3)$ \\
\hline Chemotherapy & $14(23.3)$ \\
\hline Radiotherapy and chemotherapy & $10(16.7)$ \\
\hline None & $34(56.7)$ \\
\hline \multicolumn{2}{|l|}{ Differentiation } \\
\hline G1 & $15 / 35(42.9)$ \\
\hline G2 & $19 / 35(54.3)$ \\
\hline G3 & $1 / 35(2.9)$ \\
\hline Unknown & 25 \\
\hline \multicolumn{2}{|l|}{ Lymphovascular invasion } \\
\hline Yes & $2(3.3)$ \\
\hline No & $58(96.7)$ \\
\hline \multicolumn{2}{|l|}{ Depth of cervical interstitial infiltration } \\
\hline$\leq 1 / 2$ & $38 / 53(71.7)$ \\
\hline$>1 / 2$ & $15 / 53(28.3)$ \\
\hline Unknown & 7 \\
\hline \multicolumn{2}{|l|}{ Uterine infiltration } \\
\hline Yes & $4(6.7)$ \\
\hline No & 56 (93.3) \\
\hline \multicolumn{2}{|l|}{ Lymph node metastasis } \\
\hline Yes & $3(5.4)$ \\
\hline No & $53(94.6)$ \\
\hline Unknown & 4 \\
\hline Median (IQR) follow-up (month) & $50.2(5.1-154.6)$ \\
\hline Recurrence/death & 0 \\
\hline
\end{tabular}

${ }^{*}$ Results are number (\%) unless stated otherwise.

NACT, neoadjuvant intravenous chemotherapy; ; NAIC, neoadjuvant arterial interventional chemotherapy. 
Table 2 Comparison of clinicopathological features between patients with villoglandular adenocarcinoma and those with standard cervical adenocarcinoma*

\begin{tabular}{|c|c|c|c|}
\hline & $\begin{array}{l}\text { Villoglandular adenocarcinoma } \\
(n=47)\end{array}$ & Standard adenocarcinoma $(n=104)$ & $P$ value \\
\hline Median age \pm SD & $43.8 \pm 7.6$ & $47.1 \pm 8.9$ & $0.029^{*}$ \\
\hline $\mathrm{BMI}, \mathrm{kg} / \mathrm{m}^{2}$ & $22.2 \pm 2.9$ & $23.0 \pm 2.8$ & 0.22 \\
\hline Menopause & 33 & 104 & $<0.001^{*}$ \\
\hline Yes & $27 / 33(81.8)$ & $69(66.3)$ & \\
\hline No & $6 / 33(18.2)$ & $35(33.7)$ & \\
\hline FIGO stage (2009) & & & $0.001^{*}$ \\
\hline IA1 & 0 & $13(12.5)$ & \\
\hline $\mathrm{IA} 2$ & $4(8.5)$ & $5(4.8)$ & \\
\hline IB1 & $35(74.5)$ & $83(79.8)$ & \\
\hline IB2 & $8(17.0)$ & $3(2.9)$ & \\
\hline Surgery & & & 0.79 \\
\hline Radical hysterectomy & $42(89.4)$ & $90(86.5)$ & \\
\hline Simple hysterectomy & $5(10.6)$ & $14(13.5)$ & \\
\hline \multicolumn{4}{|l|}{ Adjuvant therapy } \\
\hline Yes & $28(59.6)$ & $64(61.5)$ & 0.86 \\
\hline No & $19(40.4)$ & $40(38.5)$ & \\
\hline Grade & & & $0.003^{*}$ \\
\hline I & $13 / 28(46.4)$ & $10 / 57(17.5)$ & \\
\hline II & $14 / 28(50.0)$ & $28(49.1)$ & \\
\hline III & $1 / 28(3.6)$ & 19 (33.3) & \\
\hline Tumor size $($ mean $\pm S D)$ & $3.0 \pm 1.3$ & $2.6 \pm 1.3$ & 0.20 \\
\hline$<2 \mathrm{~cm}$ & $14(29.8)$ & 49/101 (48.5) & \\
\hline $2-4 \mathrm{~cm}$ & $21(44.7)$ & 41/106 (40.6) & \\
\hline$\geq 4 \mathrm{~cm}$ & $12(25.5)$ & 11/106 (10.9) & \\
\hline Depth of cervical interstitial infiltration & & & $0.004^{*}$ \\
\hline$\leq 1 / 2$ & $28 / 40(70.0)$ & $40 / 60(66.7)$ & \\
\hline$>1 / 2$ & $12 / 40(30.0)$ & $20 / 60(33.3)$ & \\
\hline Lymphovascular invasion & & & 0.37 \\
\hline Yes & 2/36 (5.6) & $11 / 75(14.7)$ & \\
\hline No & $34 / 36(94.4)$ & $64 / 75(85.3)$ & \\
\hline Uterine infiltration & & & 0.32 \\
\hline Yes & $3(6.4)$ & $5(4.8)$ & \\
\hline No & $44(93.6)$ & $99(95.2)$ & \\
\hline Parametrium invasion & & & 1.00 \\
\hline Yes & 0 & $1(1.0)$ & \\
\hline No & $47(100.0)$ & $103(99.0)$ & \\
\hline Lymph node metastasis & & & 0.72 \\
\hline Yes & $2 / 40(5.0)$ & $8 / 90(8.9)$ & \\
\hline No & $38 / 40(95.0)$ & $82 / 90(91.1)$ & \\
\hline Recurrence & 0 & 6 & \\
\hline $\begin{array}{l}\text { Median relapse-free survival } \\
\pm \text { SD (months) }\end{array}$ & $60.5 \pm 36.0$ & $33.4 \pm 25.4$ & $<0.001^{*}$ \\
\hline Death & 0 & 3 & \\
\hline Median survival time $\pm \mathrm{SD}$ (months) & $60.5 \pm 36.0$ & $34.5 \pm 25.6$ & $<0.001^{*}$ \\
\hline
\end{tabular}

${ }^{*}$ Results are number (\%) unless stated otherwise BMI, body mass index; SD, standard deviation.

node metastasis, lymphovascular invasion, parametrial invasion. Guo et al compared villoglandular adenocarcinoma with adenocarcinoma in patients with FIGO 2009 stage I and found lower recurrence rates in those with villoglandular adenocarcinoma ( $2.6 \%$ vs $14.6 \%, p=0.038) .{ }^{13}$ The 5 -year disease-free survival of patients with villoglandular adenocarcinoma in our study was 


\section{Original research}
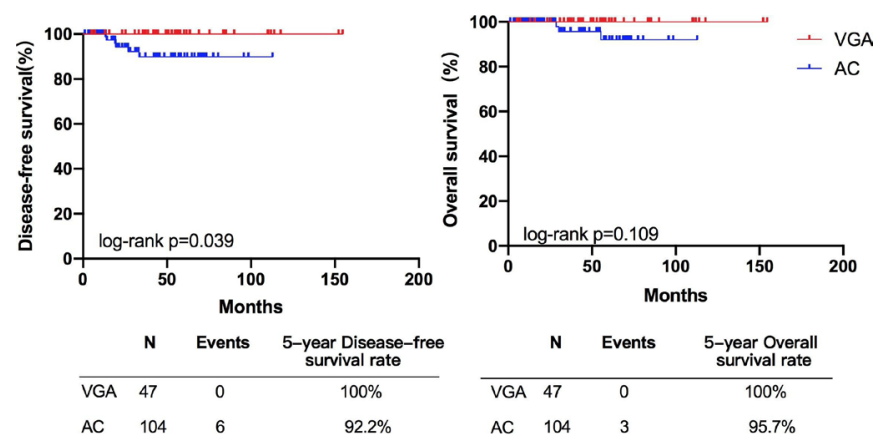

Figure 2 Comparison of survival outcomes between villoglandular adenocarcinoma and cervical adenocarcinoma. AC, adenocarcinoma; VGA, villoglandular adenocarcinoma.

also significantly better than for patients with standard cervical adenocarcinoma.

However, the less radical management approaches are still controversial. Data on conservative approaches, such as conization, simple hysterectomy, radical vaginal trachelectomy, and ovarian preservation, are limited. ${ }^{13} 1625 \mathrm{Kim}$ et al reported on three patients with villoglandular adenocarcinoma who underwent cervical conization only, and none relapsed $(I A 1=2, \mid B 1=1){ }^{12}$ Guo et al reported on two patients who underwent radical vaginal trachelectomy and pelvic lymphadenectomy, and there was no recurrence. ${ }^{13}$ Five patients in our study, including one with deep cervical stromal invasion, underwent simple hysterectomy, and none had recurrence. No consensus has been reached on ovarian preservation in young women with villoglandular adenocarcinoma. A study in Korea suggested that retaining ovarian tissue for stage IB-IIA patients with villoglandular adenocarcinoma ${ }^{24}$ might not be safe. Two patients with no risk factors developed recurrence in the adnexa and then underwent bilateral oophorectomy. ${ }^{24}$ However, none of the 15 patients (FIGO 2009 stage $\mid \mathrm{A} 2=2, \mathrm{IB} 1=7, \mathrm{IB} 2=3$, $\|A 1=2\| A 2=$,1 ) who retained their ovaries in our study had a recurrence.

As we know, the Laparoscopic Approach to Cervical Cancer (LACC) trial and other recent studies have shown that minimally invasive surgery in patients with cervical cancer has a worse oncologic outcome than open surgery. ${ }^{26-29}$ Dietl et al reviewed the literature and identified 23 patients with villoglandular adenocarcinoma who were treated by minimally invasive surgery. Four experienced recurrence and one patient died. ${ }^{30}$ Despite no recurrence

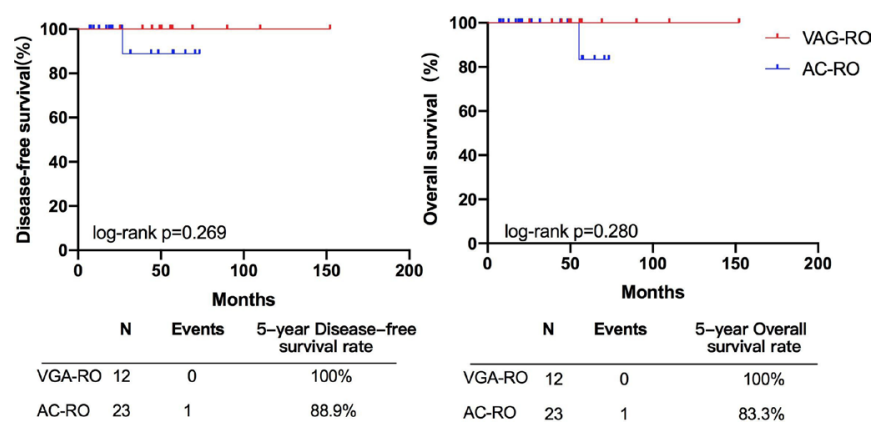

Figure 3 Comparison of survival outcomes between ovarian-reserving villoglandular adenocarcinoma and cervical adenocarcinoma. AC, adenocarcinoma; $\mathrm{RO}$, reserved ovarian-function; VGA, villoglandular adenocarcinoma. being seen in our laparoscopic surgery group, the follow-up period was shorter than for open surgery group (38.8 vs 56.5 months, $\mathrm{p}<0.001$ ).

\section{Strengths and Weaknesses}

The strengths of our study include our large sample size from multiple centers. In addition, we included patients with ovarian preservation and non-radical surgery. However, there were several limitations and these include the following. First, no central pathology review was carried out and therefore some of these tumors might have been reclassified on secondary evaluation. Second, we had no information about the criteria used for the surgical approach and post-operative management strategy. Third, during the study period, modalities of imaging and therapies might have changed within the institutions and we had no ability to control such change. Fourth, the median follow-up time of the laparoscopic surgery group was shorter than that of the open surgery group and therefore, we do not have information as to whether the laparoscopic approach affected oncologic outcomes. Lastly, as this was a multicenter study, we have no information on the auditing of data in the respective databases used in these institutions.

\section{Implications for Practice and Future Research}

The results of our study provide evidence of the indolent course of the disease in patients with cervical villoglandular adenocarcinoma. Therefore, we consider that future studies should aim to evaluate this cervical histologic sub-type as possible candidates for fertility preservation or more conservative management. Similarly, given the low rates of lymph node involvement, patients with villoglandular adenocarcinoma might need only sentinel lymph node mapping in order to assess for nodal disease.

\section{CONCLUSION}

In conclusion, villoglandular adenocarcinoma had more favorable features and better prognosis than standard adenocarcinoma. Less radical surgery or ovarian preservation might be safe for patients with early stage disease. Further investigation of the safety of these approaches is needed

\section{Author affiliations \\ ${ }^{1}$ Department of Gynecology, the First Affiliated Hospital of Wenzhou Medical University, Wenzhou, Zhejiang, China \\ ${ }^{2}$ Department of Obstetrics and Gynecology, Nanfang Hospital, Southern Medical University, Guangzhou, China \\ ${ }^{3}$ Department of Gynecologic Oncology, Shanxi Provincial Cancer Hospital, Shanxi, China \\ ${ }^{4}$ Medical Care (physical examination) Center, the First Affiliated Hospital of Wenzhou Medical University, Wenzhou, Zhejiang, China \\ ${ }^{5}$ Center for Uterine Cancer Diagnosis, Wenzhou, Zhejiang, China}

Contributors J-HC, HD, X-BY, HW-Z: protocol/project development, data analysis, and manuscript writing; $X C$ : data collection or management, data analysis; BX-L, PL, Z-QL, L-YP: data collection or management; CC, XY: protocol development, manuscript writing and editing.

Funding This work was supported by funds from the Natural Science Foundation of Zhejiang Province No. Y21H160267 (X-JY), Zhejiang Traditional Chinese Medicine Administration of China No.2020ZB144(X-JY), the Wenzhou Municipal Science and Technology Bureau of China No.Y20190014(X-JY), the National Science and Technology Support Program of China No.2014BAI05B03(C-LC), the National 
Natural Science Fund of Guangdong No.2015A030311024(C-LC), the Science and the Science and Technology Plan of Guangzhou No.158100075 (C-LC)

Competing interests None declared.

Patient consent for publication Obtained.

Provenance and peer review Not commissioned; externally peer reviewed.

Data availability statement Data are available upon reasonable request.

Supplemental material This content has been supplied by the author(s). It has not been vetted by BMJ Publishing Group Limited (BMJ) and may not have been peer-reviewed. Any opinions or recommendations discussed are solely those of the author(s) and are not endorsed by BMJ. BMJ disclaims all liability and responsibility arising from any reliance placed on the content. Where the content includes any translated material, BMJ does not warrant the accuracy and reliability of the translations (including but not limited to local regulations, clinical guidelines, terminology, drug names and drug dosages), and is not responsible for any error and/or omissions arising from translation and adaptation or otherwise.

Open access This is an open access article distributed in accordance with the Creative Commons Attribution Non Commercial (CC BY-NC 4.0) license, which permits others to distribute, remix, adapt, build upon this work non-commercially, and license their derivative works on different terms, provided the original work is properly cited, an indication of whether changes were made, and the use is noncommercial. See: http://creativecommons.org/licenses/by-nc/4.0/

ORCID iD

Xiaojian Yan http://orcid.org/0000-0002-5306-4484

\section{REFERENCES}

1 Siegel RL, Miller KD, Jemal A. Cancer statistics, 2019. CA Cancer J Clin 2019;69:7-34.

2 Ferlay J, Colombet M, Soerjomataram I, et al. Estimating the global cancer incidence and mortality in 2018: GLOBOCAN sources and methods. Int J Cancer 2019;144:1941-53.

3 Wang SS, Sherman ME, Hildesheim A, et al. Cervical adenocarcinoma and squamous cell carcinoma incidence trends among white women and black women in the United States for 1976-2000. Cancer 2004;100:1035-44.

4 Seoud M, Tjalma WAA, Ronsse V. Cervical adenocarcinoma: moving towards better prevention. Vaccine 2011;29:9148-58.

5 Mathew A, George PS. Trends in incidence and mortality rates of squamous cell carcinoma and adenocarcinoma of cervix-worldwide. Asian Pac J Cancer Prev 2009;10:645-50.

6 Galic V, Herzog TJ, Lewin SN, et al. Prognostic significance of adenocarcinoma histology in women with cervical cancer. Gynecol Oncol 2012;125:287-91.

7 Lee Y-Y, Choi CH, Kim T-J, et al. A comparison of pure adenocarcinoma and squamous cell carcinoma of the cervix after radical hysterectomy in stage IB-IIA. Gynecol Oncol 2011;120:439-43

8 Park J-Y, Kim D-Y, Kim J-H, et al. Outcomes after radical hysterectomy in patients with early-stage adenocarcinoma of uterine cervix. Br J Cancer 2010;102:1692-8.

9 Loureiro J, Oliva E. The spectrum of cervical glandular neoplasia and issues in differential diagnosis. Arch Pathol Lab Med 2014:138:453-83

10 Young RH, Scully R. Villoglandular papillary adenocarcinoma of the uterine cervix: a clinicopathologic analysis of 13 cases. Cancer 1989;63:1773-9.
11 Hodgson A, Olkhov-Mitsel E, Howitt BE, et al. International endocervical adenocarcinoma criteria and classification (IECC): correlation with adverse clinicopathological features and patient outcome. J Clin Pathol 2019;72:347-53.

$12 \mathrm{Kim} \mathrm{H}-\mathrm{J}$, Sung J-H, Lee E, et al. Prognostic factors influencing decisions about surgical treatment of villoglandular adenocarcinoma of the uterine cervix. Int J Gynecol Cancer 2014;24:1299-305.

13 Guo P, Liu P, Yang J, et al. Villoglandular adenocarcinoma of cervix: pathologic features, clinical management, and outcome. Cancer Manag Res 2018;10:3955-61.

14 Utsugi K, Shimizu Y, Akiyama F, et al. Clinicopathologic features of villoglandular papillary adenocarcinoma of the uterine cervix. Gynecol Oncol 2004;92:64-70.

15 Khunamornpong S, Maleemonkol S, Siriaunkgul S. WellDifferentiated villoglandular adenocarcinoma of the uterine cervix: a report of 15 cases including two with lymph node metastasis. $J$ Med Assoc Thai 2001;84:882-8.

16 Jones MW, Silverberg SG, Kurman RJ. Well-differentiated villoglandular adenocarcinoma of the uterine cervix: a clinicopathological study of 24 cases. Int J Gynecol Pathol 1993:12:1-7.

17 Macdonald RD, Kirwan J, Hayat K, et al. Villoglandular adenocarcinoma of the cervix: clarity is needed on the histological definition for this difficult diagnosis. Gynecol Oncol 2006;100:192-4.

18 Costa MJ, Mcllnay KR, Trelford J. Cervical carcinoma with glandular differentiation: histological evaluation predicts disease recurrence in clinical stage I or II patients. Hum Pathol 1995;26:829-37.

19 Koh WJ, Aburustum NR, Bean SM. Cervical cancer, version 3.2019. NCCN Clinical Practice Guidelines in Oncology. J Natl Compr Canc Netw 2019;17:64-84.

20 Lataifeh IM, Al-Hussaini M, Uzan C, et al. Villoglandular papillary adenocarcinoma of the cervix: a series of 28 cases including two with lymph node metastasis. Int J Gynecol Cancer 2013;23:900-5.

21 Korach J, Machtinger R, Perri T, et al. Villoglandular papillary adenocarcinoma of the uterine cervix: a diagnostic challenge. Acta Obstet Gynecol Scand 2009;88:355-8.

22 Stanley-Christian H, Heim BK, Hines JF. Villoglandular adenocarcinoma of the cervix: a report of three cases and review of the literature. Gynecol Oncol 1997;66:327-30.

23 Hopson L, Jones MA, Boyce CR, et al. Papillary villoglandular carcinoma of the cervix. Gynecol Oncol 1990;39:221-4.

24 Ju UC, Kang WD, Kim SM. Is the ovarian preservation safe in young women with stages IB-IIA villoglandular adenocarcinoma of the uterine cervix? J Gynecol Oncol 2018;29:e54.

25 Ballo MS, Silverberg SG, Sidawy MK. Cytologic features of welldifferentiated villoglandular adenocarcinoma of the cervix. Acta Cytol 1996;40:536-40.

26 Ramirez PT, Frumovitz M, Pareja R, et al. Minimally invasive versus abdominal radical hysterectomy for cervical cancer. N Engl J Med 2018;379:1895-904.

27 Cusimano MC, Baxter NN, Gien LT, et al. Impact of surgical approach on oncologic outcomes in women undergoing radical hysterectomy for cervical cancer. Am J Obstet Gynecol 2019;221:619.e1-619.e24.

28 Chen X, Zhao N, Ye P, et al. Comparison of laparoscopic and open radical hysterectomy in cervical cancer patients with tumor size $\leq 2$ cm. Int J Gynecol Cancer 2020;30:564-71.

29 Uppal S, Gehrig PA, Peng K, et al. Recurrence rates in patients with cervical cancer treated with abdominal versus minimally invasive radical hysterectomy: a multi-institutional retrospective review study. $J$ Clin Oncol 2020;38:1030-40.

30 Dietl A, Aumann K, Beckmann MW. Tumor handling of earlystage cervical cancer: a literature analysis of villoglandular adenocarcinoma of the cervix. Anticancer Res 2020;40:3049-53. 